\title{
Human Antibody Against C Domain of Tenascin-C Visualizes Murine Atherosclerotic Plaques Ex Vivo
}

\author{
Tobias von Lukowicz ${ }^{* 1-3}$, Michela Silacci*4, Matthias T. Wyss ${ }^{3,5}$, Eveline Trachsel ${ }^{4}$, Christine Lohmann ${ }^{1-3}$, \\ Alfred Buck ${ }^{3,5}$, Thomas F. Lüscher ${ }^{1-3}$, Dario Neri ${ }^{4}$, and Christian M. Matter ${ }^{1-3}$ \\ ${ }^{I}$ Cardiovascular Research, Institute of Physiology, University of Zurich, Zurich, Switzerland; ${ }^{2}$ Cardiology, CardioVascular Center, \\ Zurich University Hospital, Zurich, Switzerland; ${ }^{3}$ Center for Integrative Human Physiology, University of Zurich, Zurich, Switzerland; \\ ${ }^{4}$ Department of Chemistry and Applied Biosciences, Swiss Federal Institute of Technology, Zurich, Switzerland; and ${ }^{5}$ Nuclear Medicine, \\ University Hospital Zurich, Zurich, Switzerland
}

Targeting proteins that are overexpressed in atherosclerotic plaques may open novel diagnostic applications. The $\mathrm{C}$ domain of tenascin- $C$ is absent from normal adult tissues but can be inserted during tumor progression or tissue repair into the molecule by alternative splicing. We tested the ability of the human antibody G11, specific to this antigen, to reveal murine atherosclerotic plaques ex vivo. The antibody directed against the extra domain B of fibronectin (L19) was used as a reference. Methods: We intravenously injected ${ }^{125}$-labeled G11 or L19 antibodies into apolipoprotein E-deficient $\left(\mathrm{ApoE}^{-/-}\right)$mice and harvested the aortae 4 or $24 \mathrm{~h}$ later. En face analyses of distal aortae and longitudinal sections of the aortic arch were performed to compare antibody uptake using autoradiography with plaque staining using oil red $O$. Plaque macrophages were detected by immunohistochemistry (anti-CD68 staining). Biodistribution of injected antibodies was investigated in aortae and blood at 4 and $24 \mathrm{~h}$. Results: En face analyses revealed a significant correlation between radiolabeled $\mathrm{G} 11$ and fat-stained areas, increasing from 4 to $24 \mathrm{~h}$, with a correlation coefficient of $0.92(P<0.0001)$ and an average signal-to-noise ratio of $104: 1$ at $24 \mathrm{~h}$. Plaque imaging using L19 showed similar results $(r=0.86$; $P<0.0001$; signal-tonoise ratio, $72: 1$ at $24 \mathrm{~h}$ ). Uptake of radiolabeled antibodies in histologic sections colocalized with fat staining and activated macrophages in aortic plaques. Biodistribution analyses confirmed specific accumulation in aortic plaques as well as rapid blood pool clearance of the antibodies $24 \mathrm{~h}$ after injection. Immunofluorescence analyses revealed increased expression of tenascin and fibronectin isoforms in macrophage-rich plaques. Conclusion: The antibody G11, specific to the $\mathrm{C}$ domain of tenascin- $C$, visualizes murine atherosclerotic plaques ex vivo. In conjunction with the increased expression of the $C$ domain of tenascin- $C$ in macrophage-rich plaques, the colocalization of G11 uptake with activated macrophages, and the favorable target-to-blood ratio at $24 \mathrm{~h}$, this antibody may be useful for molecular imaging of advanced atherosclerotic plaques in the intact organism.

\footnotetext{
Received Aug. 24, 2006; revision accepted Dec. 15, 2006.

For correspondence contact: Christian M. Matter, MD, Cardiovascular Research, Institute of Physiology, Zurich University, and Cardiology, Cardiovascular Center, University Hospital Zurich, Winterthurerstrasse 190, $\mathrm{CH}-8057$ Zurich, Switzerland.

E-mail: cmatter@physiol.unizh.ch

${ }^{*}$ Contributed equally to this work.

COPYRIGHT @ 2007 by the Society of Nuclear Medicine, Inc.
}

Key Words: atherosclerosis; autoradiography; molecular imaging; cardiology (basic/technical); correlative imaging

J Nucl Med 2007; 48:582-587

DOI: 10.2967/jnumed.106.036046

$\mathbf{T}$ he vulnerable plaque poses an important problem in cardiovascular disease. Bearing the risk of rupturing, the vulnerable plaque may cause either plaque progression (1) or complete arterial lumen obstruction leading to myocardial infarction or stroke. Currently, the clinical diagnosis of atherosclerotic plaques relies on methods such as angiography, ultrasound, CT, optical coherence tomography, and MRI. These techniques give insight into plaque morphology with ever-increasing spatial resolution, but they provide only limited information about plaque biology. In principle, molecular information about processes in plaque formation and stabilization could be gained using specific ligands (typically, human monoclonal antibodies) that recognize suitable markers of disease and that are radiolabeled for detection by PET.

Atherosclerosis is increasingly recognized as a chronic inflammatory disease (2). Particularly, macrophages that become foam cells or T-lymphocytes have been shown to play a crucial role in atherogenesis (3). In accordance with this concept, we have reported that treatment of atherosclerotic mice with an antiinflammatory agent such as a c-Jun N-terminal kinase inhibitor (4) decreases atherosclerosis. Furthermore, we and others have shown that patients with systemic inflammatory diseases such as rheumatoid arthritis and systemic lupus erythematosus exhibit an increased incidence of atherosclerotic disease and myocardial infarctions (5-7).

Inflammation and tumor growth are associated with the formation of new blood vessels. The human recombinant antibody L19, specific to the extra domain B of fibronectin, has been shown to selectively localize around newly formed tumor blood vessels both in animal models and in patients with cancer $(8,9)$. A radiolabeled derivative of the L19 antibody (10) and the fusion protein between L19 and interleukin-2 (11) are currently being investigated in clinical 
trials. Furthermore, we have recently shown that radioactive or fluorescent preparations of the L19 miniantibody (or small immune protein [SIP]) revealed atherosclerotic plaques in $\mathrm{ApoE}^{-1-}$ mice (12), thus opening attractive opportunities for imaging atherosclerotic plaques or for targeted delivery of therapeutic agents.

Tenascin- $\mathrm{C}$ is a large, adhesive extracellular matrix glycoprotein that can accommodate the insertion of extra domains by a mechanism of alternative splicing during tumor progression or tissue remodeling (13). Interestingly, tenascin-C also plays an important role in vascular diseases. Indeed, tenascin-C expression is induced after balloon injury (14), in progressive pulmonary vascular disease (15) in arterialized vein grafts (16), and-notably-in macrophage-rich human coronary atherosclerotic plaques (17). Recently, we have discovered that the extra domain $\mathrm{C}$ ( $\mathrm{C}$ domain), being undetectable in normal adult tissues (18), displays a more restricted pattern of expression than do other domains. Taking advantage of a large library of synthetic human antibody phage displays, we have isolated a high-affinity miniantibody against the $\mathrm{C}$ domain of tenascin- $\mathrm{C}$ (hereafter termed G11) and showed that specific antibodies to this target were able to stain vascular structures in tumors (19) with specifically high tumor-to-blood ratios for gliomas and lung tumors $(20)$.

In this study, we have characterized expression of the $\mathrm{C}$ domain of tenascin-C within murine atherosclerotic plaques. After intravenous administration of this antibody into $\mathrm{ApoE}^{-1-}$ mice, we demonstrated the ability of radiolabeled G11 to selectively identify plaques ex vivo and to colocalize with activated plaque macrophages. Comparisons of the plaque-targeting ability of radiolabeled G11 or L19 revealed that both antibodies displayed similar signal-tonoise ratios.

\section{MATERIALS AND METHODS}

\section{Antibodies}

Both G11 and L19 were expressed in HEK293 cells in the miniantibody SIP format and were purified to homogeneity on antigen columns (10). The high-affinity G11 antibody was obtained from the parental A12 antibody $(19,20)$ using an affinity maturation procedure developed by our group (21).

\section{Animals}

Atherosclerotic plaques were analyzed in male apolipoprotein $\mathrm{E}$ knockout $\left(\mathrm{ApoE}^{-1-}\right)$ mice (22) (C57BL/6J background) that were fed, for 3-5 mo, a high-cholesterol diet (1.25\% total cholesterol, RD12108; Research Diets) that had been started at an age of $8 \mathrm{wk}$. The mice were evenly distributed between the G11 and the L19 groups. All animal experiments were performed in agreement with institutional guidelines and approved by the local animal committee.

\section{Harvest and Tissue Processing}

The mice were sacrificed 4 or $24 \mathrm{~h}$ after injection of ${ }^{125} \mathrm{I}$ labeled G11 (0.3 MBq, $8 \mu \mathrm{g} ; 12$ mice) or ${ }^{125}$ I-labeled L19 (0.3 MBq, $8 \mu \mathrm{g} ; 11$ mice) into the tail vein. We selected these time points on the basis of our previous findings using radiolabeled L19 for imaging murine atherosclerotic plaques (12). After we punctured the left ventricle and cut the right atrium, the vessels were rinsed with normal saline and the aorta was excised after dissection of the adventitial tissue. For en face analyses, the distal aorta (from left subclavian artery to iliac bifurcation) was opened longitudinally (23). For microscopic examinations, the aortic arch was embedded in optimal-cutting-temperature compound (TissueTek; Sakura) and frozen in isopentane. Serial longitudinal sections of the aortic arch (10 $\mu \mathrm{m}$ thick) were cut within $24 \mathrm{~h}$ after harvesting and thaw-mounted on glass slides for microautoradiography and immunohistochemistry. Immunofluorescence studies were performed on aortic samples of atherosclerotic $\mathrm{ApoE}^{-1-}$ mice without prior antibody injections.

\section{Plaque Imaging}

Morphology and Autoradiography. For en face macroautoradiography, the distal aortae were longitudinally opened and exposed on a phosphor-imaging screen (for $72 \mathrm{~h}$ with ${ }^{125} \mathrm{I}-\mathrm{G} 11$; for $16 \mathrm{~h}$ with ${ }^{125}$ I-L19). The area of plaque was visualized by fat staining (oil red $\mathrm{O}$ ) and photographed with a 12.5-megapixel digital camera (DP70; Olympus) that was mounted on an SZX951 microscope (Olympus Schweiz AG). For microautoradiography, longitudinal sections of aortae were exposed as above (for $7 \mathrm{~d}$ with ${ }^{125} \mathrm{I}-\mathrm{G} 11$; for $3 \mathrm{~d}$ with ${ }^{125} \mathrm{I}-\mathrm{L} 19$ ). The data were scanned (Fuji BAS $1800 \mathrm{II}$; pixel size, $50 \mu \mathrm{m}$ ) for further analyses.

Signal Quantification. The lesion-to-nonlesion (signal-to-noise) ratio in autoradiography of longitudinally opened aortae was determined using integration of signal intensity (NIH Image J 1.33) over corresponding aortic areas, taking the mean of 5 measurements from each animal. The corresponding ratios in 3 serial longitudinal sections of the proximal aorta were determined after injections of ${ }^{125} \mathrm{I}-\mathrm{G} 11$ or ${ }^{125} \mathrm{I}-\mathrm{L} 19$ by relating the signal intensity of a region of interest within the plaque to a region outside the lesion, taking the mean of 3 measurements from each animal ( $n=6$ for G11 and L19 at $24 \mathrm{~h} ; n=3$ at $4 \mathrm{~h}$ ). En face fat staining and corresponding macroautoradiography were compared by tracing all positive areas (Analysis Five Docu; SoftImaging System) and correlating the percentage of the total vessel areas positive using the Spearman rank correlation test (GraphPad Prism, version 4).

Immunofluorescence and Immunohistochemistry. Longitudinal sections of aortic arches were fixed with acetone, and fluorescence staining was performed using the following primary antibodies: SIP(L19) or SIP(G11), rat antimouse CD68 (Serotec) for macrophages, or rat antimouse CD31 (BD Pharmingen) for endothelial cells. As secondary antibodies, we used rabbit antihuman IgE (DAKO) for G11 and L19 followed by Alexa Fluor 594 goat antirabbit IgG (Molecular Probes) and, for CD68 and CD31, Alexa Fluor 488 goat antirat IgG (Molecular Probes). For immunohistochemical detection of anti-CD68 antibody, we used an alkaline phosphatase-labeled donkey antigoat antibody. Alkaline phosphatase was visualized using naphthol AS-BI phosphate and new fuchsin as substrate. Endogenous alkaline phosphatase was blocked by levamisole. Color reactions were performed at room temperature for $15 \mathrm{~min}$ with reagents from Sigma. Sections were counterstained with hematoxylin, and cover slips were mounted with glycerol and gelatin. Negative controls included omission of the first antibodies and preincubation of the first antibodies with immunogenic peptides. Slides were mounted with Glycergel mounting medium (Dako). All sections were photomicrographed with a digital camera (Olympus DP50-CU) mounted on an Olympus BX51 microscope and digitized (Analysis 5; SoftImaging System). 


\section{Biodistribution}

Radioactivity in blood and harvested aortae, liver, kidney, heart, and spleen was measured in a $\gamma$-counter (Packard Cobra II; Perkins Elmer) and expressed as percentage injected dose (\%ID) per gram of organ weight to determine biodistribution. Counting time per sample was $5 \mathrm{~min}$; decay correction was performed according to the time of injection. Blood-pool clearance between 4 and $24 \mathrm{~h}$ after injection was calculated as $100 \%(1-(\% \mathrm{ID} / \mathrm{g}$ [24 h]/\%ID/g [4 h])).

\section{Competition Assay}

To compete with the targeted antigens in vivo, the recombinant $\mathrm{C}$ domain of tenascin- $\mathrm{C}$ or the recombinant extra domain $\mathrm{B}$ of fibronectin was premixed in a 10-fold molar excess with the antibodies G11 or L19 and injected intravenously into $\mathrm{ApoE}^{-1-}$ mice. A second dose of antigen (10-fold molar excess) was injected $15 \mathrm{~min}$ later. The mice were sacrificed $24 \mathrm{~h}$ after the first injection, and aortae were processed for imaging as already described.

\section{Statistics}

Values are given as mean \pm SD. A $P$ value of less than 0.05 was considered statistically significant.

\section{RESULTS}

\section{Production and Labeling of G11 and L19 Miniantibodies with Radioiodine}

The antibodies G11 and L19 were expressed in miniantibody SIP format (Fig. 1A). Sodium dodecylsulfate-polyacrylamide gel electrophoresis of $\operatorname{SIP}(\mathrm{G} 11)$ and SIP(L19) samples demonstrated a molecular weight of $80 \mathrm{kDa}$ in nonreducing conditions and of $40 \mathrm{kDa}$ in the presence of $\beta$-mercaptoethanol (Fig. 1B), because of the reductive cleavage of an interdomain disulfide bridge between the $\mathrm{eCH} 4$ domains of human IgE, used for antibody dimerization.

\section{Selective Detection of Murine Plaques Ex Vivo with 125I-G11}

To assess plaque-targeting ability, we injected similar amounts of ${ }^{125}$ I-labeled preparations of SIP(G11) or SIP(L19) into $\mathrm{ApoE}^{-1-}$ mice and performed en face aortic autoradiography at 4 or $24 \mathrm{~h}$ after antibody injection (Fig. 2).

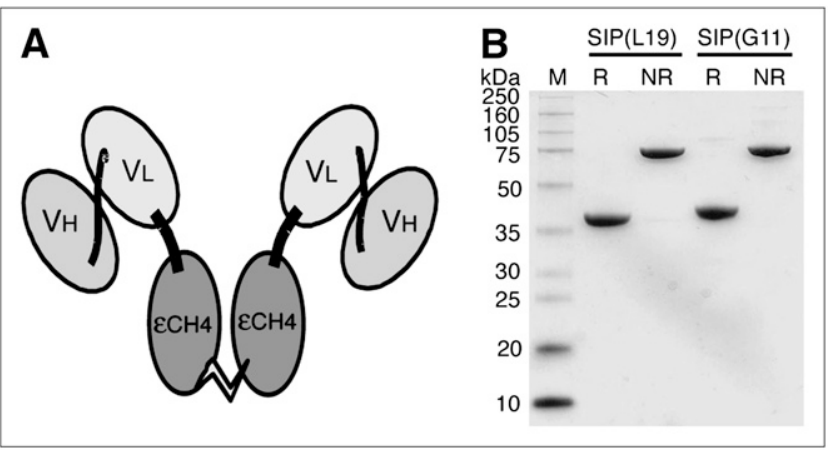

FIGURE 1. (A) Schematic structure of antibodies in SIP format. (B) Sodium dodecylsulfate-polyacrylamide gel electrophoresis analyses in reducing (R) and nonreducing (NR) conditions of G11 and L19. A pure covalent homodimer is observed for both antibodies when running the gel in nonreducing conditions.

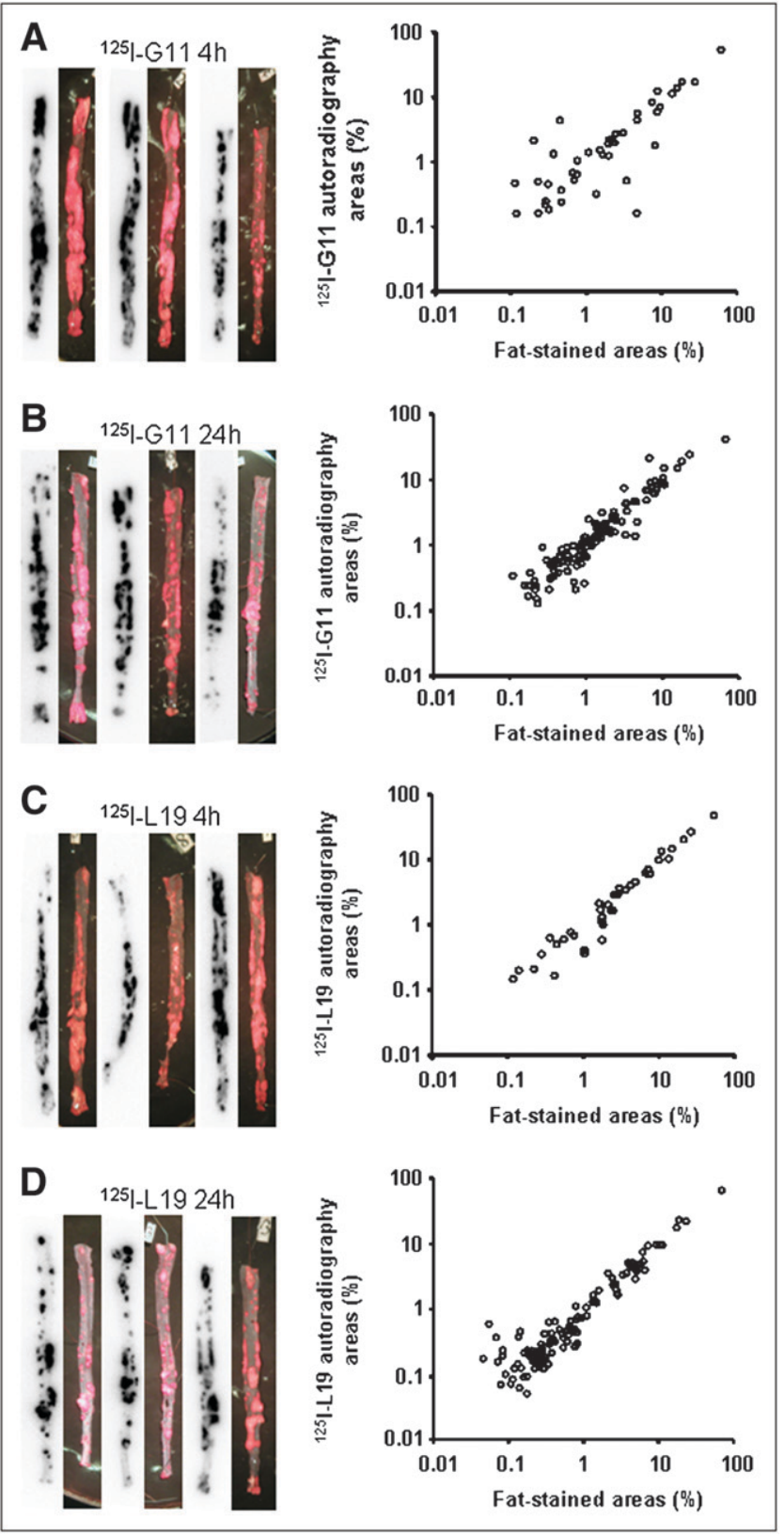

FIGURE 2. (A) Macroautoradiography (left) and fat staining using oil red $\mathrm{O}$ (right) of longitudinally opened aortae $4 \mathrm{~h}$ after intravenous injection of ${ }^{125} \mathrm{~J}-\mathrm{G} 11(0.3 \mathrm{MBq}, 8 \mu \mathrm{g}$ per animal) into apolipoprotein $\mathrm{E}$ knockout $\left(\mathrm{ApoE}^{-/-}\right)$mice $(n=4)$. Comparisons of corresponding en face autoradiographic signals and fat staining in single plaques demonstrate significant correlation $(r=0.77 ; P<0.0001)$. Each circle $(n=57)$ refers to single autoradiographic signal and its corresponding plaque staining. (B) En face analyses $24 \mathrm{~h}$ after injection of ${ }^{125} \mathrm{I}-\mathrm{G} 11$ (0.3 MBq, 8 $\mu \mathrm{g}$ per animal) into $\mathrm{ApoE}^{-1-}$ mice $(n=8): r=0.92 ; P<0.0001$; $n$ (total plaques) $=152$. (C) En face analyses $4 \mathrm{~h}$ after injection of ${ }^{125} \mathrm{I}-\mathrm{L} 19$ (0.3 MBq, $8 \mu \mathrm{g}$ per animal) into $\mathrm{ApoE}^{-/-}$mice $(n=$ 4): $r=0.93 ; P<0.0001 ; n$ (total plaques) $=50$. (D) En face analyses $24 \mathrm{~h}$ after injection of ${ }^{125} \mathrm{I}-\mathrm{L} 19(0.3 \mathrm{MBq}, 8 \mu \mathrm{g}$ per animal) into $\mathrm{ApoE}^{-1-}$ mice $(n=7): r=0.86 ; P<0.0001 ; n$ (total plaques) $=113$. 
Comparisons of the corresponding en face autoradiographic signals and fat staining in single plaques $4 \mathrm{~h}$ after injection of ${ }^{125}$ I-G11 demonstrated a highly significant correlation $(r=0.77 ; P<0.0001)$ and a sensitivity of $74 \%$ to detect fat-stained areas by autoradiography (4 mice; Fig. $2 \mathrm{~A}$ ). The mean signal-to-noise ratio of this macroautoradiography relating radioactivity uptake in plaque-bearing to plaque-free vessel wall was 43:1. Twenty-four hours after injection of ${ }^{125} \mathrm{I}-\mathrm{G} 11$, the correlation between autoradiography and fat staining increased to $0.92(P<0.0001)$, with a sensitivity of $76 \%$ (8 mice). The mean signal-to-noise ratio increased to $104: 1$ (Fig. 2B).

The corresponding analyses for ${ }^{125} \mathrm{I}-\mathrm{L} 19 \mathrm{~h}$ after injection (4 mice) revealed a similar antibody uptake (signal-tonoise ratio, 48:1; $r=0.93 ; P<0.0001$; sensitivity, $82 \%$; Fig. 2C). Comparing autoradiography and fat staining $24 \mathrm{~h}$ after ${ }^{125}$ I-L19 injection, we found a correlation of 0.86 (7 mice; $P<0.0001$; signal-to-noise ratio, $72: 1$; sensitivity, $85 \%$; Fig. 2D).

\section{Colocalization of Uptake of ${ }^{125}$ I-G11 with Plaque Macrophages}

To characterize the plaque structures targeted by SIP(G11) in more detail, we evaluated longitudinal aortic arch sections of the same $\mathrm{ApoE}^{-/-}$mice 4 and $24 \mathrm{~h}$ after injection of ${ }^{125}$ I-labeled G11 or L19, respectively (Fig. 3). Uptake of radiolabeled antibodies in adjacent histologic sections colocalized with fat staining as well as activated macrophages in aortic plaques. The signal-to-noise ratios in serial sections of the aortic arch were 50:1 for ${ }^{125} \mathrm{I}-\mathrm{G} 11$ and 50:1 for ${ }^{125} \mathrm{I}-\mathrm{L} 19$ (3 each) at $4 \mathrm{~h}$ and increased to $90: 1$ for ${ }^{125} \mathrm{I}-$ G11 and $68: 1$ for ${ }^{125}$ I-L19 at 24 h (6 each).

We did not repeat experiments with a negative control antibody, because we previously described these findings using SIP(HyHEL-10), recombinant miniantibody HyHEL10 (directed against hen egg lysozyme) (12).

\section{Accumulation of ${ }^{125} \mathrm{I}-\mathrm{G} 11$ in Atherosclerotic Aortae and Rapid Clearance from Blood}

For measuring the antibody distribution in blood, aortae, and other organs of interest, we performed biodistribution experiments of radiolabeled G11 and L19. We found an aorta-to-blood ratio of 0.45 at $4 \mathrm{~h}$ after injection of ${ }^{125} \mathrm{I}-\mathrm{G} 11$ (Table 1). This ratio tripled to 1.53 at $24 \mathrm{~h}$ after injection. The biodistribution of ${ }^{125}$ I-L19 showed a substantially slower clearance profile, resulting in both higher blood and higher organ radioactivity values. Similar to ${ }^{125} \mathrm{I}-\mathrm{G} 11$, the aorta-to-blood ratio increased from 0.38 to 3.13 between 4 and $24 \mathrm{~h}$ (Table 1). The blood-pool clearance was similar for both antibodies, with a rate of $86 \%$ from 4 to 24 $\mathrm{h}$ after injection of ${ }^{125} \mathrm{I}-\mathrm{G} 11$ and $83 \%$ for ${ }^{125} \mathrm{I}-\mathrm{L} 19$ (Table 1; 4 mice for each group). The blood clearance profiles for both G11 and L19 in these atherosclerotic mice were slower than the values measured in tumor-bearing mice $(10,19)$.

Plaque-targeting experiments with autoradiographic detection, performed by injection of radioiodinated L19 and G11 with a molar excess of the cognate recombinant antigen, led to a substantial but incomplete inhibition of plaque antibody uptake (supplemental Fig. 1; 3 for each group). The partial inhibition of plaque targeting can be explained by the fact that the 2 recombinant antigens are rapidly cleared via the renal route, with blood half-lives shorter than $20 \mathrm{~min}$.

\section{Increased Expression of C Domain of Tenascin-C in Murine Atherosclerotic Plaques}

To determine the expression pattern of the $\mathrm{C}$ domain of tenascin- $\mathrm{C}$ and extra domain $\mathrm{B}$ of fibronectin in atherosclerotic murine aortae, we performed immunofluorescence

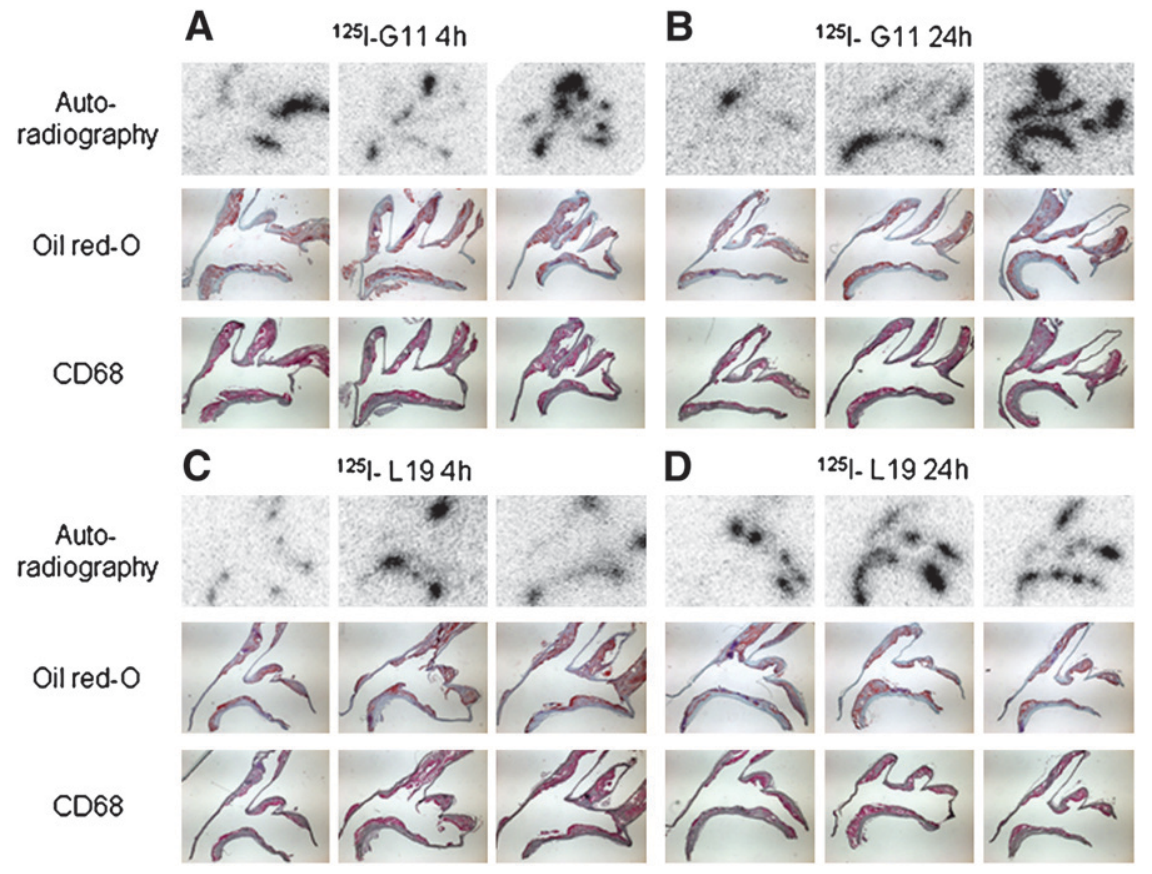

FIGURE 3. (A and B) Representative microautoradiography (top), oil red $\mathrm{O} /$ hematoxylin staining (middle), and CD68 staining (bottom) of longitudinal aortic sections $4 \mathrm{~h}(\mathrm{~A})$ or $24 \mathrm{~h}(\mathrm{~B})$ after injection of ${ }^{125} \mathrm{I}-\mathrm{G} 11$ into $\mathrm{ApoE}^{-1-}$ mice. (C and D) Corresponding micrographs at $4 \mathrm{~h}(\mathrm{C})$ or $24 \mathrm{~h}$ (D) after injection of ${ }^{125} \mathrm{I}-\mathrm{L} 19$. 
TABLE 1

Biodistribution of ${ }^{125}$ I-G11 and ${ }^{125}$ I-L19 at 4 and 24 Hours After Intravenous Injection

\begin{tabular}{|c|c|c|c|c|c|c|c|c|c|}
\hline Antibody & Time (h) & Aorta & Blood & Liver & Kidney & Heart & Spleen & Aorta/blood & $\begin{array}{c}\text { Blood } \\
\text { clearance }\end{array}$ \\
\hline G11 & 4 & $5.53 \pm 2.22$ & $12.32 \pm 2.94$ & $4.1 \pm 0.92$ & $6.73 \pm 2.09$ & $2.13 \pm 0.58$ & $5.04 \pm 1.17$ & 0.45 & \\
\hline G11 & 24 & $2.65 \pm 0.97$ & $1.73 \pm 1.16$ & $0.99 \pm 0.42$ & $0.78 \pm 0.34$ & $0.35 \pm 0.20$ & $1.18 \pm 0.84$ & 1.53 & $86 \%$ \\
\hline L19 & 4 & $11.41 \pm 4.40$ & $30.04 \pm 3.17$ & $4.67 \pm 0.77$ & $15.11 \pm 0.78$ & $6.10 \pm 0.37$ & $9.25 \pm 1.14$ & 0.38 & \\
\hline L19 & 24 & $15.97 \pm 2.29$ & $5.10 \pm 1.44$ & $1.02 \pm 0.41$ & $3.06 \pm 1.69$ & $2.13 \pm 0.69$ & $2.20 \pm 0.52$ & 3.13 & $83 \%$ \\
\hline
\end{tabular}

Aorta/blood $=\% \mathrm{ID} / \mathrm{g}$ in aorta divided by \%ID/g in blood; blood clearance $=100 \%(1-(\% \mathrm{ID} / \mathrm{g}[24 \mathrm{~h}] / \% \mathrm{ID} / \mathrm{g}[4 \mathrm{~h}]) ; n=4$ for each value. Data are mean $( \pm S D) \% I D / g$.

staining using the G11 or L19 antibodies, together with staining of macrophages and endothelial cells (Fig. 4). Both G11 and L19 stained similar plaque regions, adjacent to macrophage infiltrations and endothelial cells. L19 generally yielded a stronger immunofluorescence signal, suggesting increased antigen expression in murine macrophage-rich atherosclerotic plaques.

\section{DISCUSSION}

In this study, we showed that expression of the $\mathrm{C}$ domain of tenascin- $\mathrm{C}$ is increased in macrophage-rich plaques and that $\mathrm{G} 11$, a recombinant human antibody directed against the $\mathrm{C}$ domain of tenascin- $\mathrm{C}$, reveals murine atherosclerotic plaques ex vivo using radioiodine as a tracer.

In particular, our macroscopic findings demonstrated a good signal-to-noise ratio and a high specificity for the G11 antibody. Indeed, its signal-to-noise ratio in isolated, atherosclerotic aortae was between 54 and 104:1 and similar to that of the L19 antibody directed against the extra domain $\mathrm{B}$ of fibronectin (12). These findings in conjunction with the favorable target-to-blood ratio $24 \mathrm{~h}$ after antibody injection
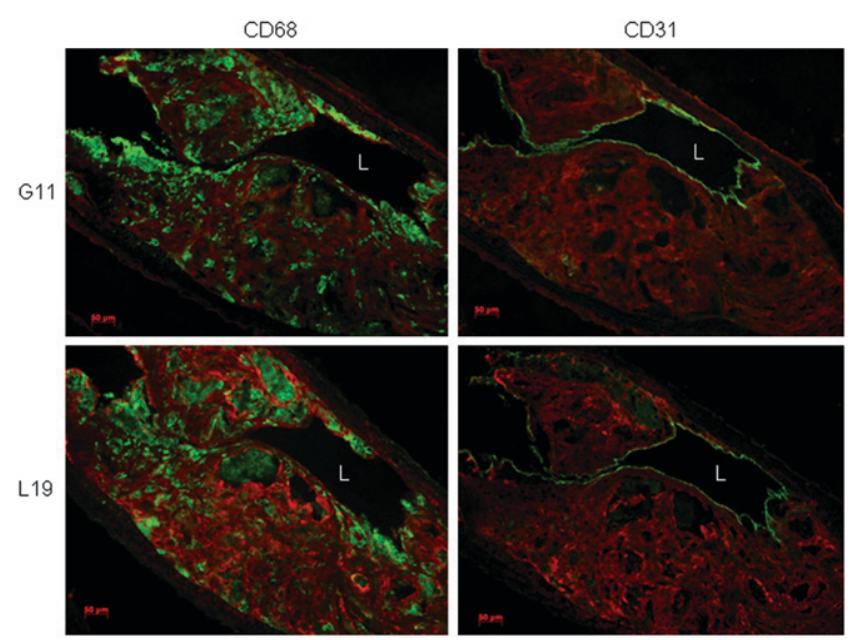

FIGURE 4. Longitudinal sections of atherosclerotic aortic arches stained for $C$ domain of tenascin-C (G11, red) or extra domain B of fibronectin (L19, red) and CD68 or CD31 (green); representative staining of 3 samples is shown. $\mathrm{L}=$ lumen; bar $=$ $100 \mu \mathrm{m}$. should set the stage for performing in vivo imaging of murine atherosclerotic plaques using PET. We anticipate overcoming the limited spatial resolution of PET by combining it with CT.

Our microscopic immunofluorescence analyses of murine plaques suggested increased antigen expression for the extra domain $\mathrm{B}$ of fibronectin, compared with the $\mathrm{C}$ domain of tenascin-C. In parallel, we needed longer exposure times for detecting the G11-derived radioactivity. Nonetheless, the high affinity of both antibodies for their cognate antigen, the fast blood clearance rate of the G11 antibody, and its low unspecific binding in normal tissue may account for the good signal-to-noise ratios observed with $\operatorname{SIP}(\mathrm{G} 11)$ in our autoradiographic studies. The more restricted staining pattern of G11 is further supported by our experience with the 2 antibodies in the tumor field: L19 is a pan-tumoral antibody, whereas G11 stains only a few cancer types-notably aggressive astrocytomas and lung cancers $(9,18)$. Furthermore, our immunofluorescence staining showed increased antigen expression of the $\mathrm{C}$ domain of tenascin- $\mathrm{C}$ and extra domain $\mathrm{B}$ of fibronectin near macrophage infiltrations. Complementary to these findings, our immunohistochemical analyses revealed colocalization of antibody staining for both G11 and L19 with activated plaque macrophages. Recently, we have shown that L19 preferentially stains advanced plaques (Thomas et al., unpublished data, 2006). Similar investigations are now justified for the G11 antibody.

Both L19 and G11 are human antibodies that recognize their murine and human antigen with high affinity.

In terms of imaging applications, the pharmacokinetic properties of the SIP antibody format nicely match the halflife of the PET tracer ${ }^{124} \mathrm{I}$. For radionuclides with a shorter half-life, smaller antibody fragments may be preferable (24). This work may also facilitate the development of targeted therapeutic compounds-for example, by conjugating the G11 antibody with suitable antiinflammatory agents.

\section{CONCLUSION}

The antibody G11, specific to the C domain of tenascin-C, reveals murine atherosclerotic plaques ex vivo. In addition, we found an increased expression of the $\mathrm{C}$ domain of 
tenascin-C in macrophage-rich plaques, a colocalization of G11 uptake with activated macrophages, and a favorable radioactivity ratio in aortic plaques to blood at $24 \mathrm{~h}$. Therefore, this antibody may be useful for molecular imaging of advanced atherosclerotic plaques in vivo.

\section{ACKNOWLEDGMENTS}

This work was funded in part by grants from the European Union G5RD-CT-2001-00532 and by Bundesamt für Bildung und Wissenschaft 02.0057, the Swiss National Science Foundation (31-114094/1, 3100-068118, and 3100A0-105804/1), the Swiss Heart Foundation, the University Research Priority Program Integrative Human Physiology at the University of Zurich, and Abbott/Jomed, Germany. Dario Neri is a consultant and shareholder of Philogen, a biotechnology company that has acquired the rights to the G11 and L19 antibodies from the Swiss Federal Institute of Technology, Zurich.

\section{REFERENCES}

1. Kolodgie FD, Gold HK, Burke AP, et al. Intraplaque hemorrhage and progression of coronary atheroma. N Engl J Med. 2003;349:2316-2325.

2. Ross R. Atherosclerosis: an inflammatory disease. N Engl J Med. 1999;340: $115-126$.

3. Libby P. Inflammation in atherosclerosis. Nature. 2002;420:868-874.

4. Ricci R, Sumara G, Sumara I, et al. Requirement of JNK2 for scavenger receptor A-mediated foam cell formation in atherogenesis. Science. 2004;306:15581561.

5. Fischer LM, Schlienger RG, Matter C, Jick H, Meier CR. Effect of rheumatoid arthritis or systemic lupus erythematosus on the risk of first-time acute myocardial infarction. Am J Cardiol. 2004;93:198-200.

6. Fischer LM, Schlienger RG, Matter CM, Jick H, Meier CR. Discontinuation of nonsteroidal anti-inflammatory drug therapy and risk of acute myocardial infarction. Arch Intern Med. 2004;164:2472-2476.

7. Asanuma Y, Oeser A, Shintani AK, et al. Premature coronary-artery atherosclerosis in systemic lupus erythematosus. N Engl J Med. 2003;349:2407-2415.
8. Santimaria M, Moscatelli G, Viale GL, et al. Immunoscintigraphic detection of the ED-B domain of fibronectin, a marker of angiogenesis, in patients with cancer. Clin Cancer Res. 2003;9:571-579.

9. Neri D, Bicknell R. Tumour vascular targeting. Nat Rev Cancer. 2005;5:436446.

10. Borsi L, Balza E, Bestagno M, et al. Selective targeting of tumoral vasculature: comparison of different formats of an antibody (L19) to the ED-B domain of fibronectin. Int J Cancer. 2002;102:75-85.

11. Carnemolla B, Borsi L, Balza E, et al. Enhancement of the antitumor properties of interleukin-2 by its targeted delivery to the tumor blood vessel extracellular matrix. Blood. 2002;99:1659-1665.

12. Matter CM, Schuler PK, Alessi P, et al. Molecular imaging of atherosclerotic plaques using a human antibody against the extra-domain B of fibronectin. Circ Res. 2004;95:1225-1233.

13. Borsi L, Carnemolla B, Castellani P, et al. Monoclonal antibodies in the analysis of fibronectin isoforms generated by alternative splicing of mRNA precursors in normal and transformed human cells. J Cell Biol. 1987;104:595-600.

14. Wallner K, Shah PK, Sharifi BG. Balloon catheterization induces arterial expression of new Tenascin-C isoform. Atherosclerosis. 2002;161:75-83.

15. Jones PL, Cowan KN, Rabinovitch M. Tenascin-C, proliferation and subendothelial fibronectin in progressive pulmonary vascular disease. Am J Pathol. 1997; 150:1349-1360.

16. Wallner K, Li C, Fishbein MC, Shah PK, Sharifi BG. Arterialization of human vein grafts is associated with tenascin-C expression. J Am Coll Cardiol. 1999;34:871-875.

17. Wallner K, Li C, Shah PK, et al. Tenascin-C is expressed in macrophage-rich human coronary atherosclerotic plaque. Circulation. 1999;99:1284-1289.

18. Carnemolla B, Castellani P, Ponassi M, et al. Identification of a glioblastomaassociated tenascin-C isoform by a high affinity recombinant antibody. Am J Pathol. 1999;154:1345-1352.

19. Silacci M, Brack S, Schirru G, et al. Design, construction, and characterization of a large synthetic human antibody phage display library. Proteomics. 2005;5: 2340-2350.

20. Silacci M, Brack SS, Spath N, et al. Human monoclonal antibodies to domain C of tenascin-C selectively target solid tumors in vivo. Protein Eng Des Sel. 2006; 19:471-478.

21. Pini A, Viti F, Santucci A, et al. Design and use of a phage display library: human antibodies with subnanomolar affinity against a marker of angiogenesis eluted from a two-dimensional gel. J Biol Chem. 1998;273:21769-21776.

22. Plump AS, Smith JD, Hayek T, et al. Severe hypercholesterolemia and atherosclerosis in apolipoprotein E-deficient mice created by homologous recombination in ES cells. Cell. 1992;71:343-353.

23. Matter CM, Wyss MT, Meier P, et al. ${ }^{18} \mathrm{~F}$-Choline images murine atherosclerotic plaques ex vivo. Arterioscler Thromb Vasc Biol. 2006;26:584-589.

24. Berndorff D, Borkowski S, Moosmayer D, et al. Imaging of tumor angiogenesis using ${ }^{99 \mathrm{~m}}$ Tc-labeled human recombinant anti-ED-B fibronectin antibody fragments. J Nucl Med. 2006;47:1707-1716. 